\title{
Erratum zu: Vorgehensweise bei der Untersuchungsplanung und Forschungsdesign
}

\section{Erratum zu:}

\section{Kapitel 2 in: A. Steffen und S. Doppler, Einführung in die Qualitative Marktforschung, essentials, https://doi.org/10.1007/978-3-658-25108-6_2}

Die originale Version dieses Buches wurde auf Seite 11 mit den beiden vertauschten Begriffen induktives versus deduktives Vorgehen publiziert. Dies ist nun korrigiert und lautet wie folgt:

Induktives Vorgehen ist im qualitativen Forschungsparadigma etabliert. Der Erkenntnisprozess beginnt mit den Daten. Es wird vom Speziellen (Erkenntnisse aus den erhobenen Daten) auf das Allgemeine (Theoriebildung) geschlossen. In einer analytischen Verallgemeinerung werden Theorien gebildet oder bestätigt, die dann in einem zweiten Schritt in quantitativer Forschung überprüft werden kann (Döring und Bortz 2016a, S. 35).

Deduktives Vorgehen ist im quantitativen Forschungsparadigma etabliert. Es wird vom Allgemeinen (Theorie) auf das Spezielle geschlossen. Der Erkenntnisprozess beginnt hier mit einer Theorie bzw. einem Modell, aus dem man empirisch überprüfbare Hypothesen ableitet. Von der Stichprobe wird dann auf

Die korrigierte Version des Kapitels ist verfügbar unter https://doi.org/10.1007/978-3-658-25108-6_2 
die Grundgesamtheit geschlossen (statistische Verallgemeinerung). Wenn die erhobenen Daten die Theorie widerlegen führt dies zu einer Kritik der Theorie; wenn die Daten die Theorie bestätigen gilt die Theorie unter der genannten Versuchsbedingungen als bestätigt (Döring und Bortz 2016a, S. 35). 